\title{
Hydrogenation of Coal
}

IN a country which had formed the habit of assessing power in terms of coal-burning in a furnace or under a boiler, the decline in the coal mining industry and the rapid expansion in the use of the internal combustion engine are two facts about which chemists and engineers have been thinking for a long time. Interest in the production of petrol from coal is now nation-wide; it has been stimulated by the hope-indeed by the removed in the form of their compounds with hydrogen, that is, as water, ammonia and hydrogen sulphide. Then there is the saturation with hydrogen of unsaturated bonds between carbon atoms. Finally, there is the splitting up by heat of the saturated molecules so formed to give light hydrocarbons, although a certain amount of hydrocarbon gas, and even of coke, is also formed, particularly when the saturation has been inade-

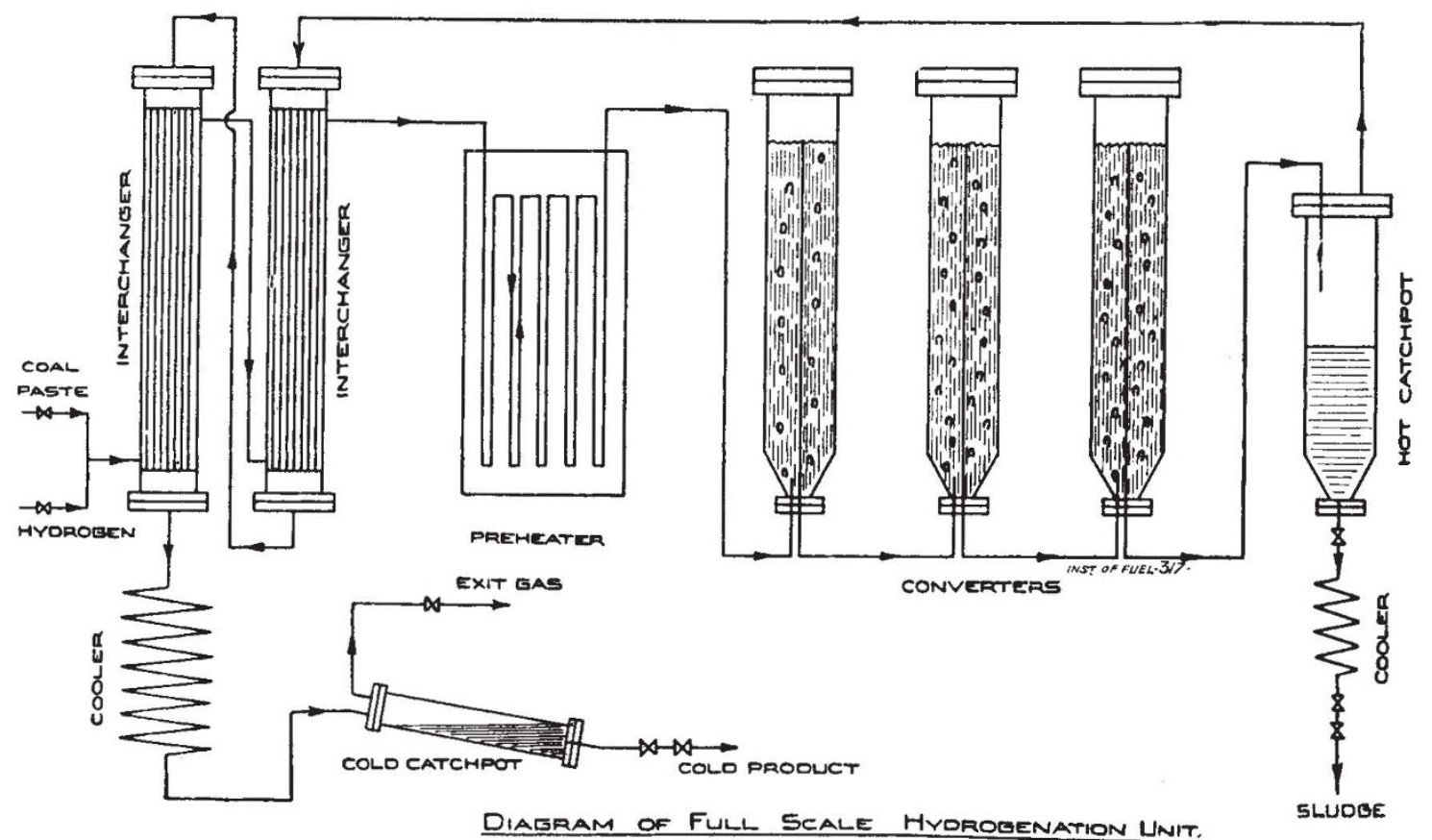

Fig. 1.

conviction - that this branch of chemical industry will appreciably help British coal mining to surmount its financial difficulties, bringing work and wages into many homes that have long known neither ; and it has been maintained by the success which Imperial Chemical Industries, Ltd., has already achieved in placing the operation on a commercially promising basis. For that reason alone, and apart from its obvious technical interest, Mr. Kenneth Gordon's lecture on November 21 to the Institute of Fuel, entitled "The Development of Coal Hydrogenation by Imperial Chemical Industries, Ltd.", deserves to be regarded as an event of more than passing significance.

Mr. Gordon reminded us that there are three types of reaction to be distinguished. First, there is the transformation of impure carbonaceous material into hydrocarbons, other elements being quate. "The art of hydrogenation," Mr. Gordon said, "consists in choosing the proper conditions of temperature, pressure, time of reaction, and catalysis, so that the three desired reactions take place to the greatest extent, and the fourth reaction, the undesired formation of gas, to the minimum extent." In practice, pressures of at least 200 atmospheres, and temperatures of $300^{\circ}-$ $500^{\circ} \mathrm{C}$. are employed, suitable means being used to dissipate the heat liberated during the chemical reaction; the catalyst may be mixed with a liquid, or vapours mixed with hydrogen may be passed over it. The choice of a catalyst is governed by its activity in promoting the hydrogenation reaction, the thermal decomposition ('cracking') reaction, or both, and its susceptibility to inactivation ('poisoning') by certain materials, for example, sulphur, often present in the original coal or oil. 
The solution of this important problem was an essential preliminary to any advance in the direction of commercial hydrogenation.

The original Bergius process, as developed by the German combine known as the Interessen Gemeinschaft fur Farbenindustrie, or more briefly as the "I.G.", working at first independently and

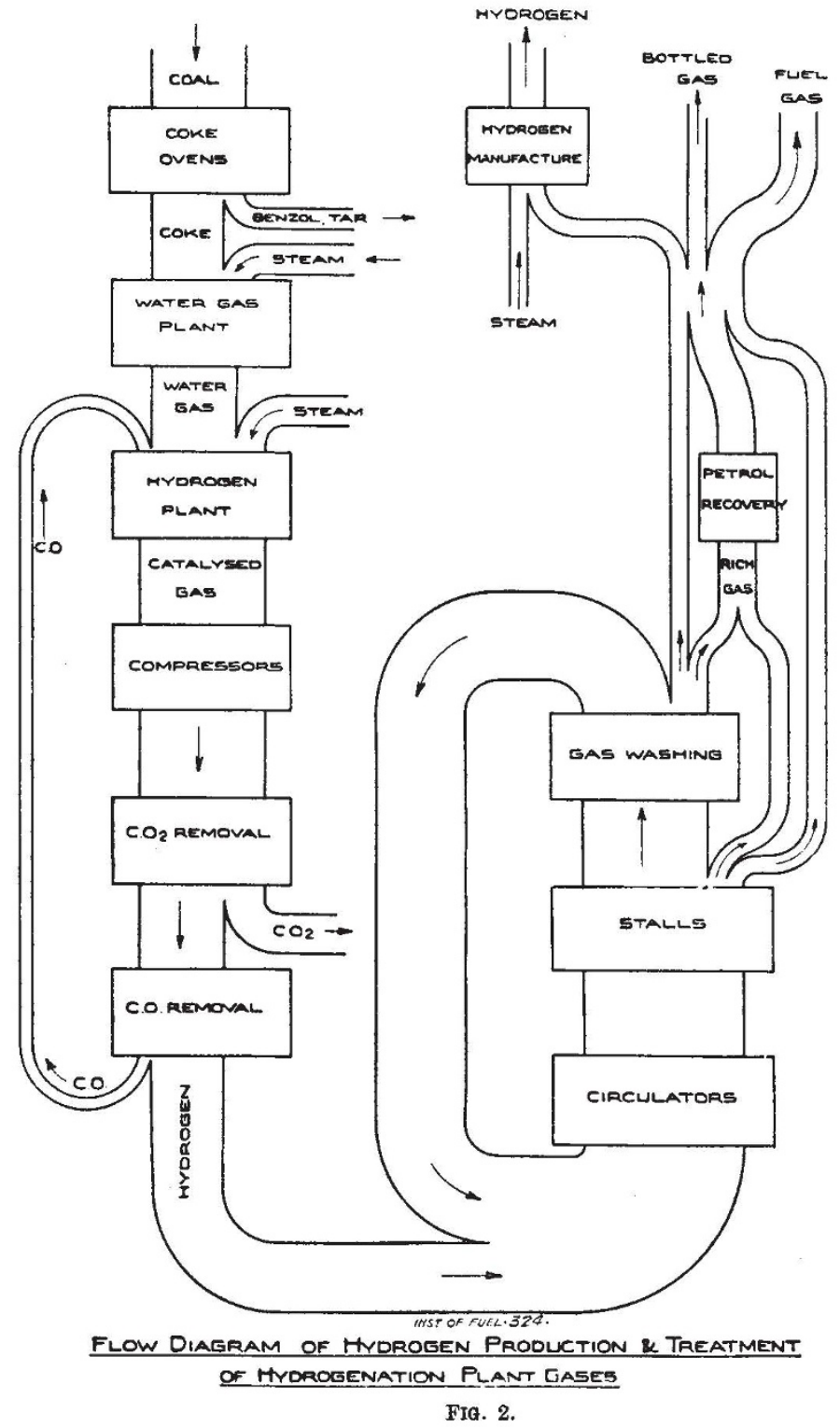

operated from 1929 until 1931. Following a demonstration in 1930 that 60 per cent by weight of petrol (a figure since exceeded) could be obtained from coal, the formation, in 1931, of the International Hydrogenation Patents Co. (a company formed by the "I.G.", "I.C.I.", the Standard Oil Co. of New Jersey, and the Royal Dutch-Shell Group) placed the interested companies in a very strong patent and technical position. During 1932 technical advances were being made, and schemes for operat ing on a large scale were maturing; so that when, in 1933, the British Government announced its intention to protect the new industry by guaranteeing the continuance of a preference for a term of years, Imperial Chemical Industries, Ltd., decided to proceed at once with the erection of a plant at Billingham.

Preliminary scientific work has, of course, been enormous in its extent; a million pounds were spent in this way between 1927 and 1933 by I.C.I. alone, but few details relating to it have appeared in the scientific literature by reason of arrangements made with the international company. It has been found possible on a large scale to dispense with mechanical stirring and external heating; tin, first used in the form of tinned iron plates, and later in the form of certain organic compounds, was found to be the most promising catalyst. The "Billingham" method of using two vessels, one inside the other, has been employed; thus one vessel withstands the temperature but not the pressure, whilst the other withstands the pressure and not the temperature.

The process affords petrol, middle oil and heavy oil ; the heavy oil is recircu. lated with coal, but the middle oil has to be subjected to hydrogenation in the state of vapour. Cleaned coal is found to be much more satisfactory than raw coal, partly because it avoids the expense of processing mere ash, but partly also because the alkaline ash interferes with the catalytic reaction. The residual ash can be neutralised by the addition of hydrolater in association with the Standard Oil Co. of New Jersey, afforded as products petrol, tar acids, heavy oil and pitch. Tests on British coals were carried out, but it was soon decided by Imperial Chemical Industries, Ltd. (equally well known as "I.C.I."), that modification in the direction of the production of petrol alone was desirable. Experiments were commenced at Billingham in 1927, and a plant treating 10 tons of coal a day was gen chloride now that its subsequent removal (in order to prevent corrosion) can be accomplished by scrubbing the hot vapours at $450^{\circ} \mathrm{C}$. with a suspension of alkali in oil.

On the large scale, the hydrogenation of coal is at present associated with that of creosote oil, supplied by tar distillers, and of low-temperature tar; but if market conditions require, small adjustments to the plant will adapt it to the desired 
extent for the treatment of coal. In fact the Billingham plant is deliberately designed to provide for flexibility. The hydrogen comes from coal by way of coke and water-gas, but it is intended to manufacture hydrogen from waste hydrocarbon gases by catalytic treatment with steam instead of using these gases as fuel. Part of the waste gas is, however, already used commercially ; liquefied butane is supplied for sale for domestic purposes where coal gas is not available.

The process is operated as follows: The coke, from coke ovens, is used for making water-gas, which is freed from sulphur, and then treated with steam in the presence of a catalyst to afford a mixture of hydrogen, carbon dioxide and a little carbon monoxide. This gas is compressed, freed from carbon dioxide by scrubbing with water at 50 atmospheres, and from carbon monoxide by scrubbing with a copper solution at 250 atmospheres pressure. The hydrogen circulating in the plant becomes contaminated with nitrogen and hydrocarbons; these are removed partly by dissolution in the products themselves (being recovered on release of pressure) and partly by scrubbing with oil under pressure.

Coal, first freed from dust by air and then cleaned by the Chance process (of floating the raw coal on a suspension of sand in water), is ground up, together with the right amounts of catalyst and oil, into a paste; the oil used for this purpose is the heavy fraction of creosote oil. This fluid paste, containing 50 per cent of coal, is injected against a pressure of 250 atmospheres, and becomes mixed with hydrogen, being brought to the reaction temperature first in heat exchangers and then in gas-fired pre-heaters. After passage through the converters the product is separated into gases and vapours on one hand and residual oil, ash and coal on the other.

The units, or 'stalls', comprise converters, heat exchangers and pre-heaters, which are arranged in one line and tended for maintenance by a Titan crane of 170 tons capacity; each pair of stalls has a control room containing the instruments and valves. The gas released from the crude products by the removal of pressure in stages is separated into 'lean' and 'rich' gas, the latter being further treated for recovery of the light fractions of petrol. All petrol is washed with caustic soda to remove hydrogen sulphide, and is stored under nitrogen ; liquid phase petrol requires treatment with sulphuric acid as well.
It is estimated that the over-all consumption of raw coal would, on a new plant, be $3 \cdot 5-4$ tons per ton of petrol; the thermal efficiency is 40 per cent, whilst that for the generation of electric power is 25 per cent, and that for gasification is 55 per cent. In the Billingham plant, the over-all raw coal consumption is 5 tons per ton of petrol.

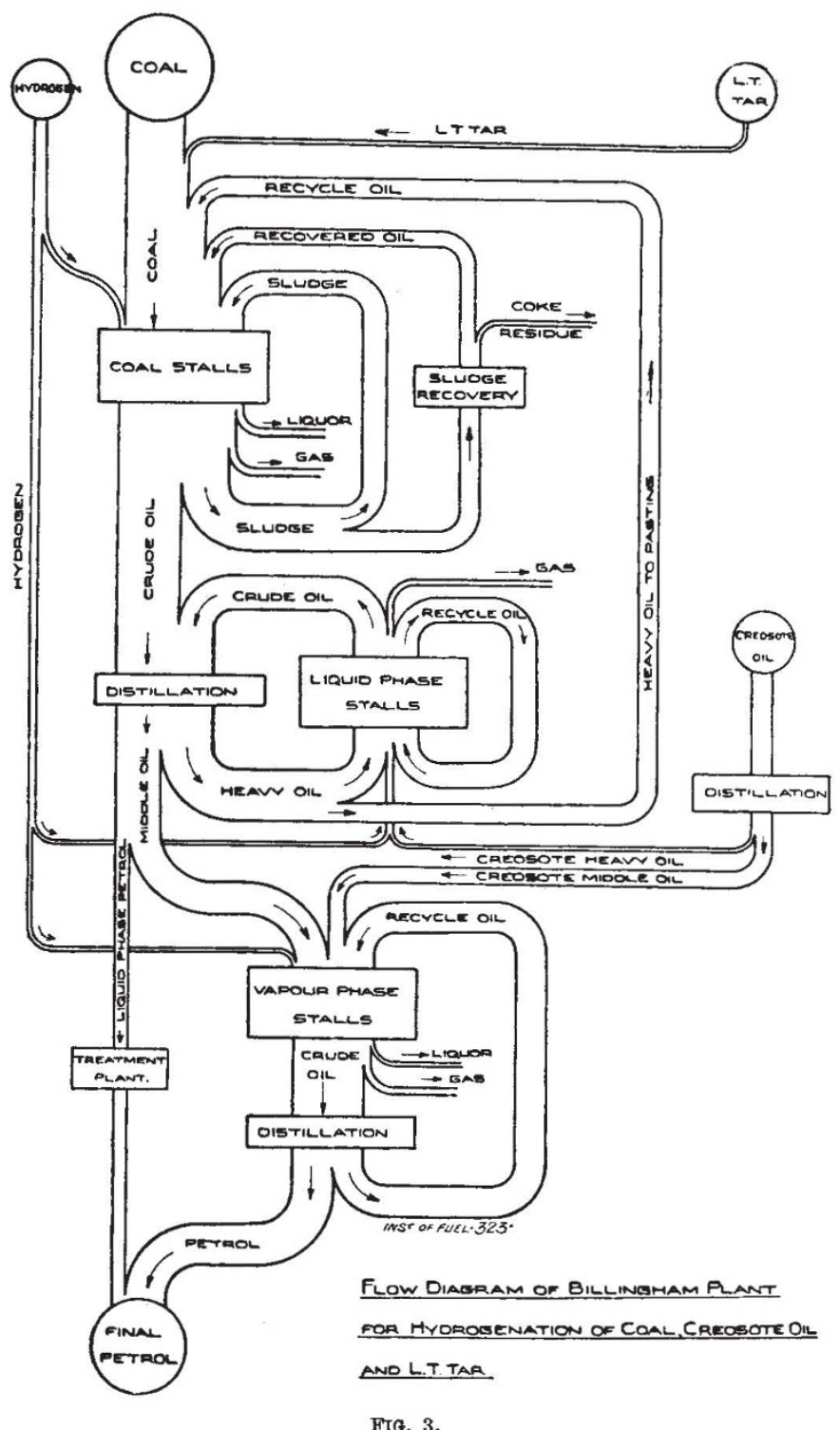

The petrol is of uniformly good quality, and has a high volatility. Concluding his paper, Mr. Gordon remarked that the company has not had much time to study the production of Diesel oil from coal, but that there is no insuperable difficulty in producing a satisfactory oil. Heavy oil obtained from coal is suitable for use as fuel oil without further treatment; whilst the problem of lubricating oil manufacture still awaits attention. 